\title{
High resolution orientation mapping of secondary phases in ATI 718Plus ${ }^{\circledR}$ alloy
}

\author{
Robert Krakow ${ }^{1}$, Mark C. Hardy ${ }^{2}$, Catherine M.F. Rae ${ }^{1, \text { a }}$, and Paul A. Midgley ${ }^{1, a}$ \\ ${ }^{1}$ Department of Materials Science and Metallurgy, University of Cambridge, 27 Charles Babbage Road, CB3 0FS \\ Cambridge, UK \\ ${ }^{2}$ Rolls-Royce plc, PO Box31, Derby DE24 8BJ, UK
}

\begin{abstract}
The polycrystalline superalloy ATI 718Plus $®$ (hereafter 718Plus) has been developed to replace the established alloy Inconel 718 by offering higher temperature capability for applications in gas turbines. The alloy exhibits two secondary phases in the austenitic matrix; it is strengthened by the $\gamma^{\prime}$-phase with $\eta$-phase discontinuously precipitated at the grain boundaries. It can be utilized to control grain growth during forging. Generally, hexagonal $\eta$ phase has been reported to possess a defined crystallographic orientation with the matrix. However, the material studied here exhibits blocky $\eta$-phase that has been precipitated and grown during thermomechanical processing. Therefore a measurable change in orientation relationship is expected. The standard technique for orientation mapping is electron back-scattered diffraction with spatial resolution of $100 \mathrm{~nm}$. That is insufficient for studying $\eta$-phase in 718Plus. By applying high resolution orientation mapping in the transmission electron microscope (Philips CM 300 FEGTEM) equipped with a Nanomegas ASTAR ${ }^{\mathrm{TM}}$ system a resolution of $3 \mathrm{~nm}$ was achieved. The indexed diffraction data was analysed using the Matlab Toolbox Mtex. The analysis included grain reconstruction and exclusion of low confidence measurements. The data set allows generating phase boundary maps indicating interfaces characteristics. Quantitative assessment shows that only $19 \%$ of the $\gamma-\eta$-interfaces fulfil the orientation relationship.
\end{abstract}

\section{Introduction}

Nickel base alloys are a complex class of materials with up to $40 \mathrm{wt} \%$ of added alloying elements. The intricate chemistry facilitates their application at high homologous temperatures by providing the basis for different strengthening mechanisms [1]. Precipitate strengthening is probably the most important mechanism utilizing coherent secondary phases [2-4]. The aim of this study is to be able to discern phase boundaries of different types in order to be able to quantify them and to understand their influence on mechanical properties and materials behaviour in the long term [5].

The superalloy 718Plus is an excellent candidate to study phase boundaries since it exhibits multiple secondary phases and different morphologies depending on thermo-mechanical history of the material [6]. One example is the boundary between the matrix phase and disc-shaped $\eta$ phase in 718Plus. The hexagonal $\eta$ phase can occur as fine colony of needles or in a more blocky morphology, compared to the first. While the blocky $\eta$ can be studied using conventional electron backscattered electron diffraction, this is not possible for the thin lamellar morphology. The ASTAR ${ }^{\mathrm{TM}}$ system allows reliable mapping of nm-sized objects $[7,8]$.

${ }^{a}$ Corresponding authors: Catherine M.F. Rae - cr18@cam. ac.uk, Paul A. Midgley - pam33@cam.ac.uk

\section{Acquisition conditions}

The phase and orientation mapping experiments were conducted on a Philips CM300 FEGTEM with retrofitted ASTAR $^{\mathrm{TM}}$ system (NanoMEGAS). The optimal acquisition conditions depend on the specimen material and preparation and should be adjusted to deliver diffraction patterns which can be recognized unambiguously by the indexation software. For the herein discussed 718Plus sample from forged and heat treated material the parameters in Table 1 were found to work best.

Detailed description of the ASTAR ${ }^{\mathrm{TM}}$ system can be found elsewhere [9-12]. In brief, a map is generated from a site of interest which is scanned using a focussed electron beam. The generated diffraction pattern is recorded for each point of the scanned area and is subsequently compared to a set of diffraction pattern templates in a semiautomatic process that yields the orientation and phase information for each pixel.

The condenser aperture is essential in defining the collection angle of the beam at the condenser lens. For the mapping experiment an aperture size must be chosen that allows a high beam current for a satisfactory signal to noise ratio.

The step and spot size need to be chosen such that the map resolution can be used to reveal details of the features studied. The spot size defines the area (volume) of excited material for each pixel. The step size is the distance been 
Table 1. TEM/ASTAR acquisition parameters for high resolution orientation mapping of 718Plus specimens.

\begin{tabular}{|l|c|}
\hline Parameter & $\begin{array}{c}\text { Recommended } \\
\text { value }\end{array}$ \\
\hline Acceleration voltage, $\mathrm{kV}$ & 300 \\
\hline Condenser aperture, $\mu \mathrm{m}$ & 50 \\
\hline Spot size, $\mathrm{nm}$ & 10 \\
\hline Camera Length L, cm (as per TEM screen) & 15 \\
\hline Step size, $\mathrm{nm}$ & 12.5 \\
\hline Precession angle $\left(^{\circ}\right)$ & 0.5 \\
\hline
\end{tabular}

individual scans. Careful consideration is required to avoid double or under sampling. A step size of $3 \mathrm{~nm}$ delivered acceptable results, however $7 \mathrm{~nm}$ were preferred due to the shorter acquisition times.

\subsection{Precession electron diffraction (PED)}

The Digistar unit is used for the scanning of the area of interest. In addition, it can be used to apply precession electron diffraction (PED), first used by Vincent and Midgley in 1993 [13]. For PED the electron beam is tilted and rotated at a constant angle around the optical axis above the specimen to give a hollow cone illumination. This is compensated by an equal and opposite action below the specimen. Combined, the double-conical scanning is equivalent to the precession of the sample.

With PED enabled the Ewald sphere intersects a larger fraction of possible reflections. In consequence reflections further out in reciprocal space are excited than would be the case for a fixed illumination. Precession therefore leads to a reduction of dynamical diffraction, thus the intensity of spots is closer to the kinematical values. Both effects are beneficial for the matching process between the recorded diffraction patterns and the templates. Two examples are given in Fig. 1.

\subsection{Camera speed}

The diffraction spot patterns are recorded using a high speed camera at a suitable exposure time to collect all excitations of the rotating electron beam.

The position of the camera relative to the phosphorous screen defines the warping of the image recorded by the camera and needs to be kept constant. This is important for the post-acquisition analysis which involves the dewarping of the recorded image of the diffraction spot pattern.

\subsection{Post-acquisition analysis}

The ASTAR ${ }^{\mathrm{TM}}$ software package includes a tool that allows the analysis of the acquired data set. Individual pixels can be selected and the diffraction pattern studied. But more importantly, virtual apertures can defined that lead to virtual dark-field maps. These maps can highlight areas of similar crystallographic properties and can therefore help to assess the quality of the acquisition process.

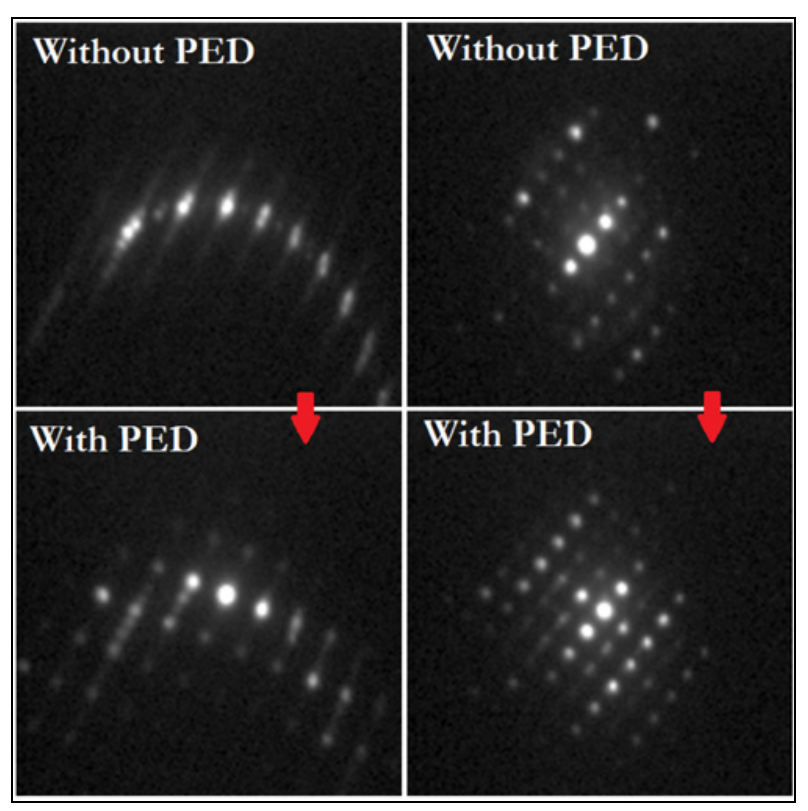

Figure 1. Effect of PED on 718Plus diffraction patterns, less dynamical diffraction and reflections excited further out in reciprocal space; Top: two examples of diffraction patterns acquired without PED and Bottom: Same positions, diffraction patterns acquired with PED.

\section{Indexation procedure}

\subsection{Template banks}

Basic crystallographic information of known phases (space group, unit cell parameters, and atom motif) is used to simulate diffraction patterns. In addition, the TEM parameters used; acceleration voltage and spot size can also be defined, all of which influence a diffraction pattern during the experiment. Full details are given in Table 2. The software generates a bank of templates covering reciprocal space with a resolution depending on the number of templates chosen. 5000 templates were reported to equal a resolution of $1^{\circ}$ for hexagonal material [14]; 2000 templates to $1^{\circ}$ angular resolution for cubic material [10]. Each template represents a set of three unique Euler angles and therefore orientation information. The process of template generation has to be repeated for each expected or possible phase.

The disc-shaped lamellar phase in 718Plus, that first forms at grain boundaries during common forging and heat treatment conditions, has repeatedly been reported to be orthogonal delta phase [15-19]. We found that the discontinuous phase is in fact hexagonal $\eta$ phase and generated templates accordingly.

\subsection{Image correction}

The fact that the diffraction pattern is projected onto the phosphorous screen before the high speed camera records it means that it is distorted and has to be corrected digitally. The correction can be performed in the ASTAR $^{\mathrm{TM}}$ software pack. For the set up used here two corrections are necessary; a horizontal and a vertical by minus 18 and minus 72 degrees (correlating to factors of 
Table 2. Analysis conditions for semi-automatic pattern matching (indexation).

\begin{tabular}{|l|c|}
\hline $\begin{array}{l}\text { Setting } \\
\text { type }\end{array}$ & $\begin{array}{c}\text { Recommended } \\
\text { value }\end{array}$ \\
\hline Camera Length L, cm (analysis parameter) & 16.35 \\
\hline Horizontal distortion & 18 \\
\hline Vertical distortion & 62 \\
\hline Number of templates (eta) & 5151 \\
\hline Number of templates (nickel) & 1326 \\
\hline
\end{tabular}

1.07619 and 1.55556), respectively. The other important value for the image correction is camera length. During the course of the experiment it was found that there can be a notable difference between camera length values as displayed in the TEM and the recorded image.

\subsection{Matching process}

During the matching process the recorded image of each scanned point is compared to all templates of each template bank. A matching function defines the similarity between template and pattern and attributes a value, called the confidence index. The highest confidence index found is automatically chosen to be the best solution for that pixel of the scanned area. The confidence index is thus a measure for the quality of the matching process and can be used to improve the subsequent generation of orientation and phase maps similar to the MAD (mean angular deviation) in other software solutions.

The second quality measure is the reliability index. It symbolizes the uniqueness of the best matching result. The value will be high if only one good solution has been found. It will be lower if the second best solution is almost as good as the best.

\subsection{Data processing}

For the generation of orientation and phase maps as well as maps for confidence and reliability index the Matlab toolbox Mtex [20,21] was used. The data transfer was done via the .ang export format.

The quality values can be used in order to clean up the data set. During this process incorrectly indexed points can be eliminated. Due to the setup of the indexation process there is no pixel which is left unindexed.

\section{Results}

The clean data set can further be processes. Classical disorientation angles are meaningful for describing grain boundary characteristics with the same crystal system $[22,23]$. For the case of hexagonal eta phase $\left(\mathrm{P} 6_{3} / \mathrm{mmc}\right)$ [24] which can precipitate with a known orientation relationship (Blackburn orientation relationship) in cubic gamma matrix (Fm3m) [25], there is no predefined solution available in Mtex. However, an algorithm was programmed that highlighted those phase boundaries that fulfilled the Blackburn orientation relationship (1).

$$
\begin{gathered}
\{11 \overline{1}\} \gamma \|(0001) \eta \\
\langle 1 \overline{1} r\rangle \gamma \|(2 \overline{1} \overline{1} 0) \eta .
\end{gathered}
$$

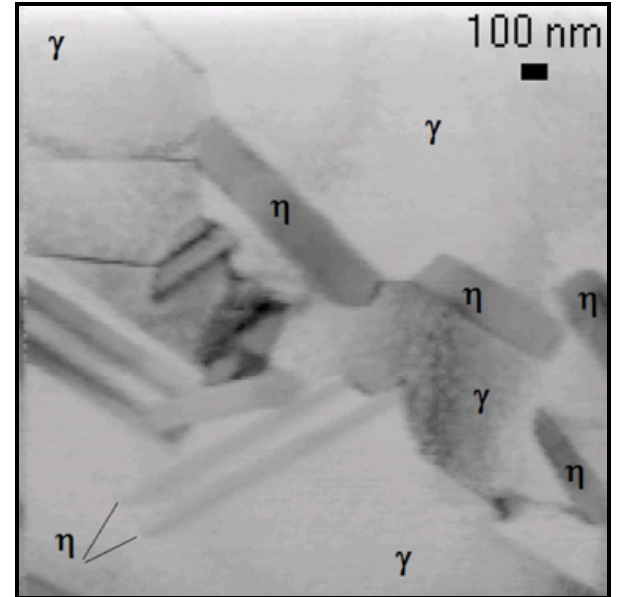

Figure 2. Virtual bright field image of complex 718Plus microstructure showing the analysed area of interest with several eta particles $(\eta)$ surrounded by gamma matrix $(\gamma)$. Several phase not annotated to aid clarity.

\subsection{Semi-quantitative results}

High resolution orientation mapping was applied to a representative area of $2237.5 \times 2237.5 \mathrm{~nm}^{2}$ of 718 Plus forged microstructure. The area was selected based on the occurrence of several precipitates that partially resided at grain boundaries, partially in the grain interior (see Fig. 2). The precipitates were several $100 \mathrm{~nm}$ in length and ranged from 50 to $300 \mathrm{~nm}$ in diameter. A separate paper describing the disc shape of the $\eta$ phase is in preparation.

Figures $3 \mathrm{~A}$ and $\mathrm{B}$ represent maps of quality values, reliability and confidence index, for the acquired data set. Both maps were generated using a similar colour code with dark blue values correlating to low and yellow/red correlating to high quality values. When looking at them in detail it can be concluded that reliability values drop at grain and phase boundaries, mainly due to overlaying diffraction patterns of either side of the boundary. Moreover, low reliability values can be found for orientations that are less unique.

A useful way of depicting a data set is to plot phase map and orientation maps. In Fig. 3E eta is depicted in light green and gamma is shown in light purple. A phase map is clearly easier interpretable then a bright field image, with varying brightness values from very bright to very dark, depending on their orientation. A virtual bright field image is given in Fig. 2. The orientation map can also give useful insight into the forged microstructure. Annealing twins, known to occur in nickel alloys, can thus more easily be identified than tilting onto a suitable diffraction condition. In order to study the specific orientation relationship of eta phase and matrix, the Mtex toolbox was used to combine boundary maps.

Firstly, phase boundaries were identified using the phase information in the data set. Secondly, a unique disorientation angle was found, that represents the Blackburn orientation relationship and a disorientation angle plot was generated, highlighting these boundaries. 

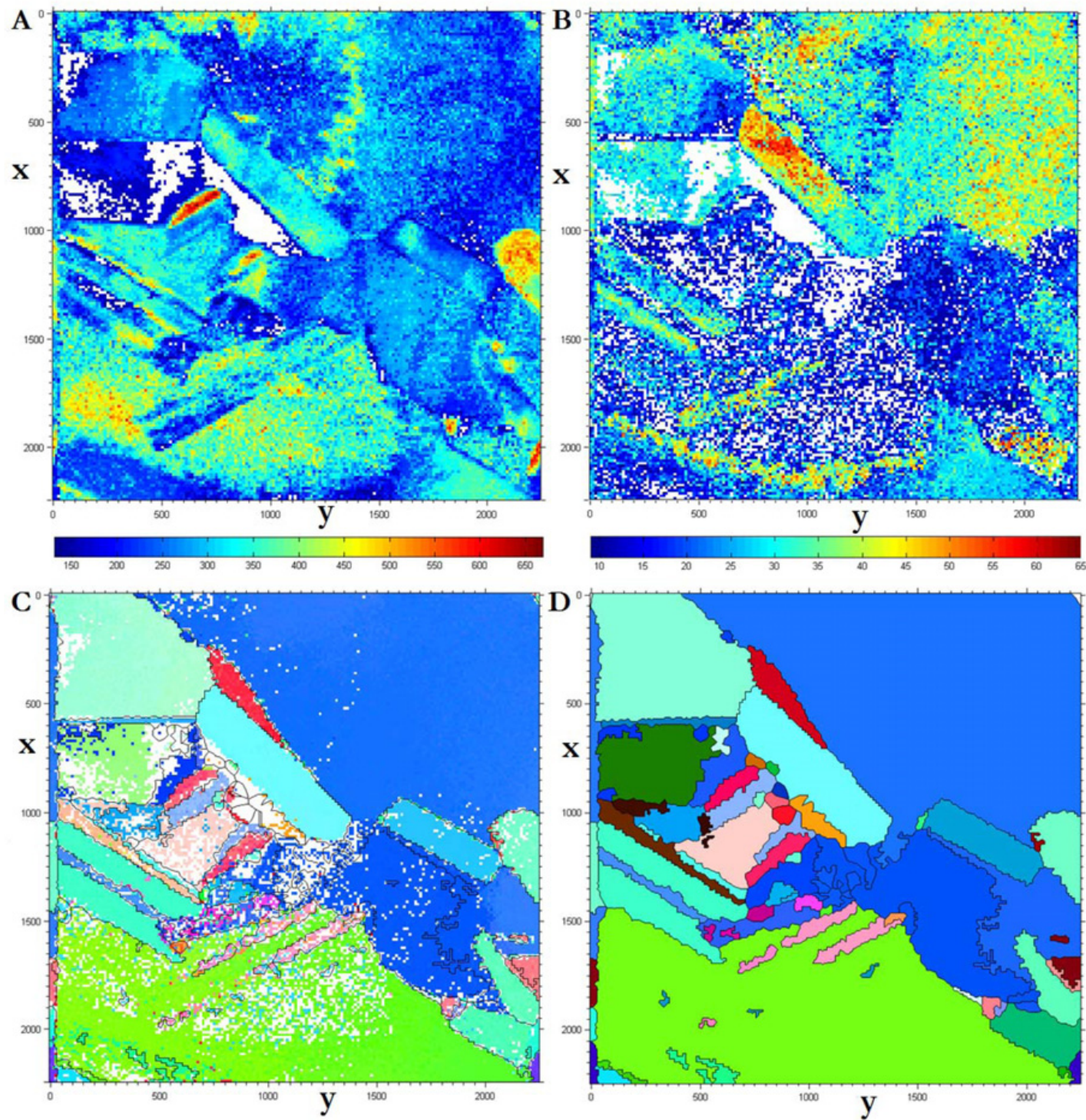

Figure 3. Representative 718Plus forged microstructure with several eta particles. A) Map of confidence index for values above 130. B) By using A as source: Map of reliability index for values above 8, high confidence in yellow and red, low in blue. C) map depicting HROM raw data. D) orientation map indicating grain orientation.

A combined plot (Fig. 3F) highlights boundaries that most likely represent boundaries with Blackburn orientation relationship. Finally, for the limited number of boundaries the validity of the results were checked and confirmed. The software furthermore allows the readout of boundary length. For the depicted data set in Fig. 3 it was found that $19 \%$ of boundaries fulfil the Blackburn orientation relationship. It is important to note that this does not mean that $19 \%$ of eta phase are lamellar rather than blocky since blocky eta phase can also have one or more phase boundaries fulfilling the requirements.

This analysis can be a first step in understanding the effect of discontinuously precipitated phase at grain boundaries on fatigue crack propagation. Coherent eta phase residing in the cubic gamma matrix is likely to exhibit stronger phase boundaries and a low misfit between the phases, making them more desirable than high misfit boundaries. For a more applied discussion the location of the phase also needs to be considered. If discontinuous eta phase, for example, has grown into the grain interior and does not cover the grain boundary, it cannot act as potential barrier for intergranular cracks. These considerations are not covered in this approach.

The authors acknowledge and thank Rolls-Royce plc for their financial support in the completion of this work. Permission to publish this article has been given by Rolls-Royce Deutschland and the University of Cambridge. The authors would like to thank Dr A.S. Eggeman and Dr J. Potillo for their assistance during the project. This work was supported by Rolls-Royce and the EPSRC under EP/H022309/1 and EP/H500375/1. We would like to thank Nanomegas for the provision of the ASTAR system under the auspices of EC FP7 grant ESTEEM2. 

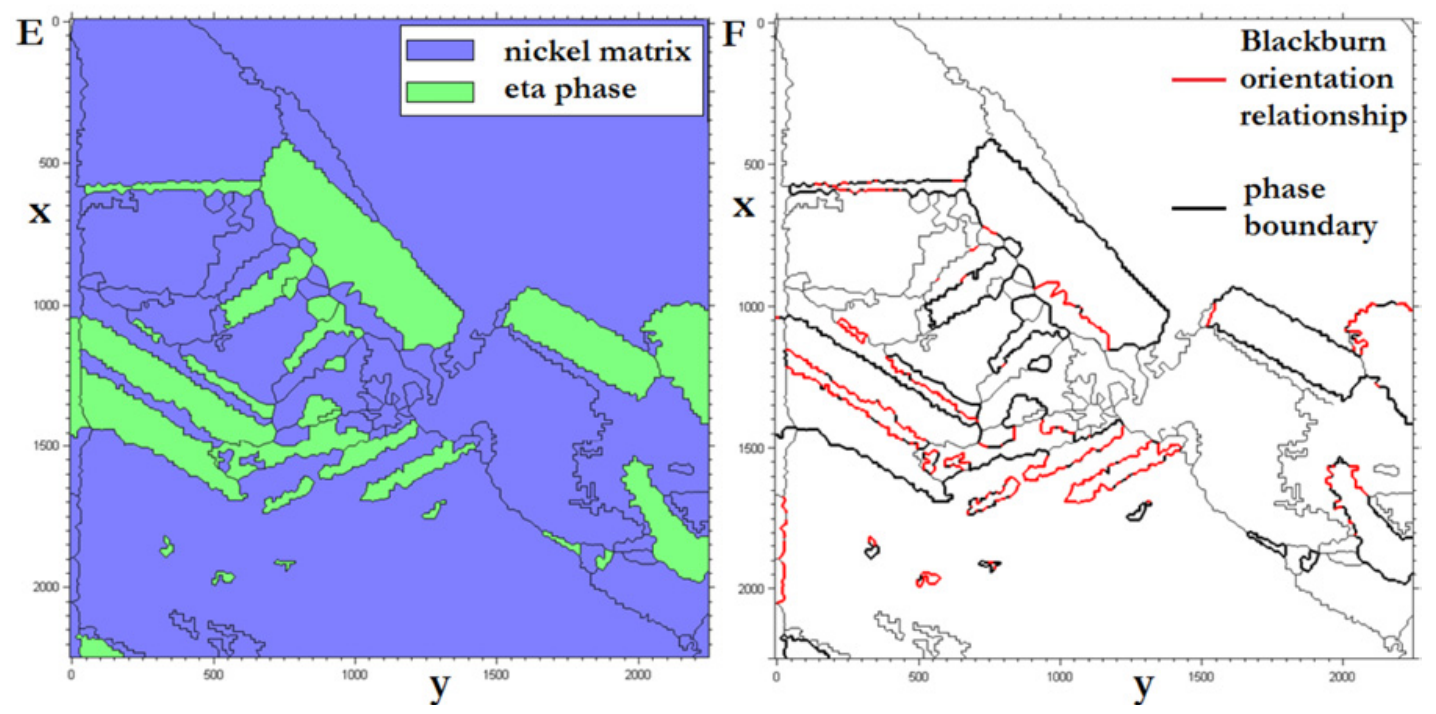

Figure 3. (Continued.) Representative 718Plus forged microstructure with several eta particles. E) Phase map, nickel matrix in blue, eta phase in green. F) Phase boundary map. In red: phase boundary with Blackburn orientation relationship.

The investigations in this paper are furthermore related to a research programme on 718 Plus, conducted at Rolls-Royce Deutschland, with funding by the Bundesministerium für Wirtschaft und Technologie (BMWi) under grant number $20 \mathrm{~T} 0813$.

\section{References}

[1] R. W. Kozar, A. Suzuki, W. W. Milligan, J. J. Schirra, M. F. Savage, and T. M. Pollock, Metall. Mater. Trans. A, 40, 1588-1603, (2009)

[2] J. W. Brooks and P. J. Bridges, Superalloys 1988, 3342, (1988)

[3] R. Cozar and D. A. Pineau, Met. Trans., 4, 47-59, (1973)

[4] G. A. Zickler, R. Radis, R. Schnitzer, E. Kozeschnik, M. Stockinger, and H. Leitner, Adv. Eng. Mater., 12, 176-183, (2010)

[5] M. Springmann, W. Rothkegel, D. Huenert, and H. Schlums, 7th Intern. Conf. LCF, 1-8, (2013)

[6] K. Lhnert, Doktorarbeit (PhD thesis), Universitt Erlangen-Nürnberg, (2011)

[7] X. Liu, N. T. Nuhfer, a. D. Rollett, S. Sinha, S.B. Lee, J. S. Carpenter, J. E. LeDonne, et al., Acta Mater., 64, 333-344, (2014)

[8] A. Kobler, A. Kashiwar, H. Hahn, and C. Kübel, Ultramicr., 128, 68-81, (2013)

[9] E. F. Rauch, M. Véron, J. Portillo, D. Bultreys, Y. Maniette, and S. Nicolopoulos, Microsc. Anal. Nanotechn. Suppl., 22, 5-8, (2008)

[10] E. F. Rauch, S. Rouvimov, S. Nicolopoulos, and P. Moeck, Microsc. Microanal., 15, 756-757, (2009)

[11] E. F. Rauch, J. Portillo, S. Nicolopoulos, D. Bultreys, S. Rouvimov, and P. Moeck, Zeitschrift für Krist., 225, 103-109, (2010)
[12] P. Moeck, S. Rouvimov, E. F. Rauch, M. Véron, H. Kirmse, I. Häusler, W. Neumann, D. Bultreys, Y. Maniette, and S. Nicolopoulos, Cryst. Res. Technol., 46, 589-606, (2011)

[13] R. Vincent and P. A. Midgley, Ultramicr., 53, 271282, (1994)

[14] S. Estradé, J. Portillo, J. Mendoza, I. Kosta, M. Serret, C. Müller, and F. Peiró, Micron, 43, 910915, (2012)

[15] K. Lhnert and F. Pyczak, 7th Int. Symp. Superalloy 718 Deriv., 877-891, (2010)

[16] L. Viskari and K. Stiller, Ultramicr, 111, 652-658, (2011)

[17] L. Viskari, Y. Cao, M. Norell, G. Sjöberg, and K. Stiller, Mater. Sci. Eng. A, 528, 2570-2580, (2011)

[18] C. Stotter, C. Sommitsch, J. Wagner, H. Leitner, I. Letofsky-Papst, and M. Stockinger, Int. J. Mater. Res., 4, 376-380, (2008)

[19] R. R. Unocic, K. A. Unocic, R. W. Hayes, G. S. Daehn, and M. J. Mills, 7th Int. Symp. Superalloy 718 Deriv., 607-615, (2010)

[20] F. Bachmann, R.Hielscher, H. Schaeben, Ultramicr., 111, 1720-1733, (2011)

[21] F. Bachmann, R. Hielscher, and H. Schaeben, Solid State Phenom., 160, 63-68, (2010)

[22] J. K. Mackenzie, Acta Metall., 12, 223-225, (1964)

[23] J. K. Mackenzie, Biometrika, 45, 229-240, (1958)

[24] E. J. Pickering, H. Mathur, a. Bhowmik, O. M. D. M. Messé, J. S. Barnard, M. C. Hardy, R. Krakow, K. Lhnert, H. J. Stone, and C. M. F. Rae, Acta Mater., 60, 2757-2769, (2012)

[25] R. C. Reed, The Superalloys, Fundamentals and Applications, Cambridge University Press. (2006) 